\title{
O papel da ictioterapia no tratamento da psoríase: relato de caso
}

Helena Cabral,* Joana Carneiro*

\begin{abstract}
RESUMO
Introdução: A psoríase é uma das dermatoses mais frequentes, afetando entre 1 a $3 \%$ da população portuguesa. Apresenta uma evolução crónica, com lesões que podem atingir grande parte da superfície corporal. Devido à frequente exuberância das lesões, a doença é muitas vezes percebida como estigmatizante pelo indivíduo que se sente envergonhado e rejeitado pelo outro. Pode apresentar impacto significativo nas relações sociais, na autoimagem e na autoestima. Atualmente, o tratamento consiste em reduzir o número e gravidade das lesões, podendo ser utilizadas medidas gerais, fototerapia, terapêutica tópica e/ou sistémica. Algumas destas modalidades terapêuticas podem estar contraindicadas, perder a eficácia, causar efeitos adversos sérios ou mesmo ser inacessíveis, o que faz com que o doente procure muitas vezes alternativas terapêuticas. Apesar da escassa literatura relacionada, a ictioterapia (utilizando peixes de água doce - Garra rufa) tem sido discutida como opção de tratamento. Atualmente existem diversos centros de tratamento disponíveis em Portugal, merecendo atenção por parte dos profissionais de saúde. Descrição do caso: Doente sexo masculino, 35 anos de idade, marinheiro. Apresenta psoríase vulgar em placas, do tipo grave, diagnosticada na infância. É seguido em consulta de dermatologia e de psicologia. Apresenta lesões em cerca de $60 \%$ da superfície corporal, refratárias a múltipla terapêutica instituída, mantendo apenas tratamento tópico. De referir tratamento sistémico recente com acitretina, com bom resultado durante o tratamento, mas recorrência das lesões assim que o suspendeu. Quando recorreu à consulta na Unidade de Saúde Familiar (USF) mostrava-se desanimado com o atual estado clínico e desacreditado face às possíveis terapêuticas futuras. Refere ter tido contacto com a ictioterapia num centro comercial e mostrou-se interessado em experimentar. Após 5 sessões de ictioterapia (em tanque com submersão do corpo inteiro), com aplicação tópica simultânea de calcipotriol e betametasona, constatou-se redução da escama característica das lesões e diminuição do prurido. Apresentava também melhoria significativa do humor, estando satisfeito com os resultados provisórios do tratamento. Comentário: A ictioterapia poderá ser útil nos quadros refratários e desfigurantes de psoríase vulgar. O presente caso revelou que esta modalidade, embora sedenta de evidência robusta, pode ser igualmente considerada dado o possível benefício com a sua utilização. Por outro lado, parece ser segura e inócua. Até ao momento, há apenas um número limitado de relatos de doentes que podem ter sido infetados com a exposição a G. rufa.
\end{abstract}

Palavras-chave: Psoríase; Peixes; Terapia Assistida por Animais.

\section{INTRODUÇÃO}

A psoríase é uma das dermatoses mais frequentes na dermatologia, afetando entre 1 a $3 \%$ da população portuguesa, cerca de 250 mil indivíduos. Dada a sua cronicidade, o médico de família é, na maioria das vezes, aquele que mais contacto tem com esta doença. As suas manifestações são variáveis, podendo cursar com lesões que atingem grande parte da superfície corporal, causando descon-

* Médicas Internas de Medicina Geral e Familiar USF Garcia de Orta, ACeS Porto Ocidental forto ao doente. Devido à frequente exuberância destas lesões, a doença é muitas vezes percebida como estigmatizante pelo indivíduo que se sente envergonhado e rejeitado pelo outro. Pode apresentar impacto significativo nas relações sociais, na autoimagem e na autoestima. Não havendo cura, o tratamento consiste, principalmente, em reduzir o número e gravidade das lesões, podendo ser utilizadas medidas gerais (hidratação, helioterapia...), fototerapia, tratamento tópico (corticosteroides, calcipotriol, tacrolimus) ou tratamento sistémico (metotrexato, acitretina, ciclosporina, 
imunobiológicos). ${ }^{1}$ Apesar dos diferentes tratamentos disponíveis, as lesões tendem a recorrer, levando a que o doente se encontre frequentemente insatisfeito. A ictioterapia (utilizando Garra rufa - peixes de água doce originários da Turquia), entre várias terapêuticas alternativas, tem sido discutida como uma opção, conciliada aos restantes tratamentos mais convencionais. ${ }^{2-3} \mathrm{Os}$ peixes Garra rufa alimentam-se da pele descamativa, não afetando a pele saudável, levando a uma rápida redução das escamas características desta doença e que tanto incomodam os doentes.

Apesar de pouco estudada em Portugal, está disponível em locais tão acessíveis como centros comerciais, atraindo a atenção de doentes que procuram alternativas para uma patologia que pode ser bastante desfigurante e que afeta de forma significativa a qualidade de vida. ${ }^{4}$

\section{DESCRIÇÃO DO CASO}

Trata-se de um doente do género masculino, com 35 anos de idade, solteiro, marinheiro de profissão. Obeso, sem outros antecedentes de relevo, apresentava psoríase vulgar em placas, do tipo grave, diagnosticada na infância. Mantém vigilância em consulta de dermatologia desde o diagnóstico e, mais recentemente, é seguido em consulta de psicologia. Apresenta um histórico vasto de tratamentos, designadamente fármacos de uso tópico com eficácia parcial. Tinha recusado fototerapia por incompatibilidade laboral. Apesar de passar grandes períodos em alto mar, admitindo temporadas de maior exposição solar, o doente mostrava pouca melhoria das lesões. No consultório apresentava recorrentemente lesões eritematosas, cobertas por escamas, em grande parte da superfície corporal, apesar de manter tratamento tópico com calcipotriol e betametasona de forma continuada. Recentemente tinha realizado tratamento sistémico com acitretina, com melhoria clínica durante o período de tratamento, mas recorrência das lesões assim que suspendeu o fármaco por receio de desenvolver efeitos laterais a nível metabólico. Mantinha, à data da consulta, apenas tratamento tópico com calcipotriol e betametasona.

Quando recorreu a consulta na Unidade de Saúde Familiar (USF) mostrava-se desanimado com o atual estado clínico e desacreditado face às possíveis terapêuticas futuras. Confessa ter faltado à última consulta de dermatologia por acreditar não haver solução, após tantos tratamentos frustrados. Numa passagem por um centro comercial, o doente relata ter tido conhecimento de um centro de ictioterapia, publicitada como opção de tratamento para a psoríase. Questionou a médica de família quanto à sua utilidade, vantagens e desvantagens e mostrava-se interessado em experimentar. À data da consulta apresentava lesões eritematosas cobertas de escamas no tronco, metade inferior do dorso, face externa dos membros superiores e região anterior dos membros inferiores (Figura 1).

Após cinco sessões de ictioterapia (em tanque, com submersão do corpo inteiro), e mantendo a aplicação tópica de calcipotriol e betametasona, voltou a consulta na USF. Constatava-se redução franca da escama esbranquiçada que recobria anteriormente as placas eritematosas a nível da face externa dos braços, tronco, dorso e membros inferiores (Figura 2). O doente referia também diminuição acentuada do prurido. No entanto, a alteração mais significativa era a melhoria de humor, mostrando-se o doente muito satisfeito com os resultados provisórios do tratamento e com a comodidade e facilidade com que o mesmo era feito, o que não tinha vindo a acontecer com os outros tratamentos que tinha experimentado.

\section{COMENTÁRIO}

Cerca de $20-30 \%$ dos doentes com psoríase têm formas moderadas ou graves, não controláveis de modo satisfatório por tratamentos tópicos. As terapêuticas

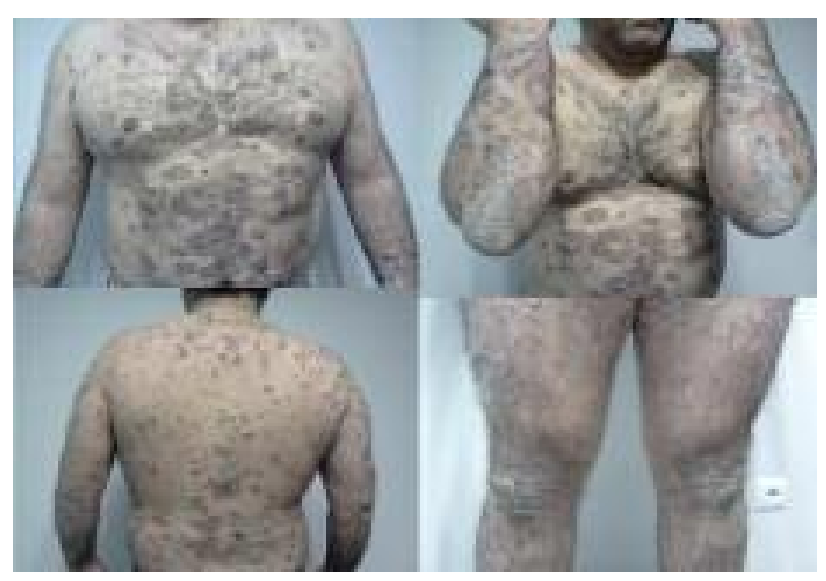

Figura 1. Lesões eritematosas, cobertas de escamas, antes das sessões de ictioterapia. 


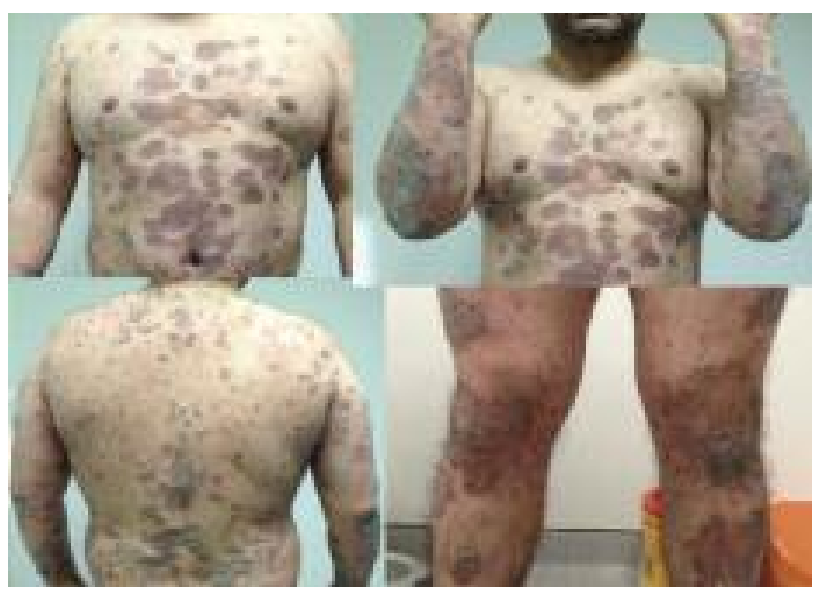

Figura 2. Lesões após cinco sessões de ictioterapia, com redução franca das escamas.

sistémicas clássicas - PUVA terapia oral, retinóides, ciclosporina e metotrexato - podem induzir toxicidade específica de órgão e uma percentagem considerável de doentes são resistentes, intolerantes ou têm contraindicações para as iniciarem. Mais recentemente estão disponíveis novos medicamentos, denominados agentes biológicos, especificamente direcionados aos mecanismos/vias envolvidos na patogénese da psoríase. A segurança destes novos medicamentos é relativamente limitada, desconhecendo-se o seu perfil de segurança a longo-prazo, além de que os seus custos financeiros são muito elevados. ${ }^{5}$

A ictioterapia poderá ser útil nos quadros refratários e desfigurantes de psoríase vulgar. Apesar de alternativa, parece ser segura e inócua. Os riscos relatados associados à exposição a G. rufa parecem ser baixos. Até ao momento, há apenas um número limitado de relatos de doentes que podem ter sido infetados por esta via de exposição. ${ }^{6}$ Infelizmente, pouco se sabe sobre os tipos de bactérias e outros potenciais microrganismos implicados neste tipo de tratamento. ${ }^{6}$ Estes relatos, no entanto, levantam algumas preocupações, nomeadamente, no que concerne ao controlo da água e transporte dos peixes, que poderão abrigar agentes patogénicos responsáveis por zoonoses de relevância clínica. Este risco pode, provavelmente, ser reduzido pela certificação dos peixes, criados em instalações controladas sob elevados padrões de vigilância analítica. Sob esta perspetiva, pacientes com determinadas morbilidades, como diabetes mellitus ou imunossupressão, devem ser desencorajados a realizar tais tratamentos.

O presente caso retrata que as terapêuticas alternativas poderão ser também consideradas, apesar da escassa literatura disponível. O facto de se revelar como terapêutica com rápida resposta, aliada a importante efeito positivo no humor do doente, devem merecer especial atenção ao profissional de saúde.

Importa ainda não descurar os restantes tratamentos. É essencial que a prescrição terapêutica seja adequada às expectativas dos doentes, considerando todos os recursos existentes, ou seja, as terapêuticas convencionais. Além disso, dever-se-á ainda ponderar a utilização das novas terapêuticas (imunobiológicos), respeitando as orientações formais existentes.

Ficam algumas questões em aberto: será que um tratamento tipicamente realizado em ambiente termal na Turquia terá o mesmo efeito em Portugal, em centros comerciais? Será suficiente a presença dos Garra rufa? É de realçar, no entanto, a opinião extremamente positiva deste doente, fazendo sentido considerar esta alternativa, de preferência em associação com tratamentos mais convencionais. Não se pode, no entanto, menosprezar a escassez de estudos relativos ao tema e a possibilidade de risco de infeção veiculada por estes peixes.

\section{REFERÊNCIAS BIBLIOGRÁFICAS}

1. Quintanilla Gutiérrez E, Serrano Collantes D. Dermatologia básica em medicina familiar. Lisboa: LIDEL; 2011. ISBN 9789727576296

2. Grassberger M, Hoch W. Ichthyotherapy as alternative treatment for patients with psoriasis: a pilot study. Evid Based Complement Alternat Med. 2006;3(4):483-8.

3. Sayili M, Akca H, Duman T, Esengun K. Psoriasis treatment via doctor fishes as part of health tourism: a case study of Kangal Fish Spring, Turkey. Tourism Manag. 2007;28(2):625-9.

4. Ginsburg IH. Psychological and psychophysiological aspects of psoriasis. Dermatol Clin. 1995;13(4):793-804.

5. Marques Pinto G, Filipe P. Normas de boa prática para o tratamento da psoríase em placas em idade não pediátrica com biológicos [Guidelines for high-quality use of biologic therapies in adults with plaque psoriasis]. Acta Med Port. 2012;25(2):125-41. Portuguese

6. Health Protection Agency. Guidance on the management of the public health risks from fish pedicures. London: HPA; 2011 Aug 31 [cited 2012 Mar 21]. Available from: https://www.gov.uk/government/uploads/system/uploads/attachment_data/file/322420/Fish_Spa_guidance.pdf

\section{CONFLITOS DE INTERESSE}

As autoras declaram não ter conflito de interesses. 
ENDEREÇO PARA CORRESPONDÊNCIA

Helena Cabral

Rua Pinho Leal, $n^{\circ} 29$ - 4150-620 Porto

E-mail: helena.cabral@gmail.com
Recebido em 11-02-2014

Aceite para publicação em 08-12-2014

\section{ABSTRACT}

\section{THE ROLE OF ICTHYOTHERAPY IN THE TREATMENT OF PSORIASIS: A CASE REPORT}

Introduction: Psoriasis is a common condition affecting 1 to $3 \%$ of the Portuguese population. It has a chronic course and lesions may cover the whole surface of the skin. With obviously visible lesions, the disease is seen as stigmatizing and patients may feel embarrassment and rejection by others. It can affect social contacts, self-image, and self-esteem. Treatment aims at reducing the number and severity of skin lesions using phototherapy, topical treatment and systemic medications. Some treatments may be contraindicated, some may lose efficacy, some cause serious adverse effects, while others may not be readily available. All this may cause patients to seek alternative therapies. Icthyotherapy, using the freshwater fish Garra rufa, has been discussed as an option. This treatment is currently available in several places in Portugal and deserves the attention of health professionals.

Case description: A 35 year-old sailor with severe psoriasis diagnosed in childhood is described. He was treated by a dermatologist and a psychologist. He presented with lesions covering $60 \%$ of the skin surface, resistant to multiple treatments, and was receiving only topical therapy. He had recently been given systemic acitretin with good results during treatment but with recurrence of lesions when treatment ended. When he came to the family health unit he was discouraged by his current condition and doubtful about the possibilities for future treatment. He mentioned that he had seen icthyotherapy in a shopping centre and expressed interest in trying it. After five sessions of icthyotherapy, with total immersion of the body in a water tank and simultaneous application of calcipotriol and betamethasone, he noted a reduction in scaling and itching. He also noted an improvement in his mood and was satisfied with the early results of treatment.

Comments: Icthyotherapy may be useful in refractory and disfiguring cases of psoriasis. This case also demonstrates benefits of its use. It appears to be safe. There are a few reports of cases of patients with skin infections from exposure to $\mathrm{G}$. rufa.

Keywords: Psoriasis; Fishes; Animal Assisted Therapy. 